\title{
Hybrid projection algorithms for approximating fixed points of asymptotically quasi-pseudocontractive mappings
}

Shin Min Kang ${ }^{a}$, Sun Young Cho ${ }^{b, *}$, Xiaolong Qin ${ }^{c}$

${ }^{a}$ Department of Mathematics and RINS, Gyeongsang National University, Jinju 660-701, Korea.

${ }^{b}$ Department of Mathematics, Gyeongsang National University, Jinju 660-701, Korea.

${ }^{c}$ Department of Mathematics, Hangzhou Normal University, Hangzhou 310036, China.

Dedicated to George A Anastassiou on the occasion of his sixtieth birthday

Communicated by Professor R. Saadati

\begin{abstract}
The purpose of this paper is to modify Ishikawa iterative process to have strong convergence without any compact assumptions for asymptotically quasi-pseudocontractive mappings in the framework of real Hilbert spaces.
\end{abstract}

Keywords: Asymptotically pseudocontractive mapping; asymptotically nonexpansive mapping; fixed point; hybrid projection algorithm.

2010 MSC: 47H09, 47J25.

\section{Introduction and Preliminaries}

Throughout this paper, we always assume that $H$ is a real Hilbert space with inner product $\langle\cdot, \cdot\rangle$, and norm $\|\cdot\|$. Assume that $C$ is a nonempty closed convex subset of $H$ and $T: C \rightarrow C$ is a nonlinear mapping. We use $F(T)$ to denote the set of fixed points of $T$.

$T$ is said to be nonexpansive if

$$
\|T x-T y\| \leq\|x-y\|, \quad \forall x, y \in C .
$$

\footnotetext{
${ }^{*}$ Corresponding author

Email addresses: smkang@gnu.ac.kr (Shin Min Kang ), ooly61@yahoo.co.kr (Sun Young Cho ), qxlxajh@163.com (Xiaolong Qin )
} 
$T$ is said to be asymptotically nonexpansive [3] if there exists a sequence $\left\{k_{n}\right\} \subset[1, \infty)$ with $\lim _{n \rightarrow \infty} k_{n}=$ 1 such that

$$
\left\|T^{n} x-T^{n} y\right\| \leq k_{n}\|x-y\|, \quad \forall x, y \in C, n \geq 1 .
$$

$T$ is said to be asymptotically quasi-nonexpansive if $F(T) \neq \emptyset$ and (1.1) holds for every $x \in C$ but $y \in F(T)$. We remark here that the class of asymptotically nonexpansive mappings was introduced by Goebel and Kirk; see [3] for more details. They proved that, if $C$ is a nonempty bounded closed convex subset of a uniformly convex Banach space $E$, then every asymptotically nonexpansive self-mapping $T$ on $C$ has a fixed point. Further, the set $F(T)$ of fixed points of $T$ is closed and convex.

$T$ is said to be pseudocontractive if

$$
\langle T x-T y, x-y\rangle \leq\|x-y\|^{2}, \quad \forall x, y \in C .
$$

$T$ is said to be asymptotically pseudocontractive if there exists a sequence $\left\{k_{n}\right\} \subset[1, \infty)$ with $\lim _{n \rightarrow \infty} k_{n}=$ 1 such that

$$
\left\langle T^{n} x-T^{n} y, x-y\right\rangle \leq k_{n}\|x-y\|^{2}, \quad \forall x, y \in C .
$$

We remark here that the class of asymptotically pseudocontractive mappings was introduced by Schu; see [16] for more details.

It is clear that (1.2) is equivalent to

$$
\left\|T^{n} x-T^{n} y\right\|^{2} \leq\left(2 k_{n}-1\right)\|x-y\|^{2}+\left\|\left(I-T^{n}\right) x-\left(I-T^{n}\right) y\right\|^{2}, \quad \forall x, y \in C .
$$

The class of asymptotically pseudocontractive mappings contains properly the class of asymptotically nonexpansive mappings as a subclass, which can be seen from the following example.

Example. ([15]) For $x \in[0,1]$, define a mapping $T:[0,1] \rightarrow[0,1]$ by

$$
T x=\left(1-x^{\frac{2}{3}}\right)^{\frac{3}{2}} .
$$

Then $T$ is asymptotically pseudocontractive but it is not asymptotically nonexpansive.

$T: C \rightarrow C$ is said to be asymptotically quasi-pseudocontractive if $F(T) \neq \emptyset$ and there exists a sequence $\left\{k_{n}\right\} \subset[1, \infty)$ with $\lim _{n \rightarrow \infty} k_{n}=1$ such that

$$
\left\langle T^{n} x-p, x-p\right\rangle \leq k_{n}\|x-p\|^{2}, \quad \forall x \in C, p \in F(T) .
$$

It is clear that (1.4) is equivalent to

$$
\left\|T^{n} x-p\right\|^{2} \leq\left(2 k_{n}-1\right)\|x-p\|^{2}+\left\|x-T^{n} x\right\|^{2}, \quad \forall x \in C, p \in F(T) .
$$

In 1991, Schu [16] proved the following results for asymptotically pseudocontractive mappings in the framework of Hilbert spaces.

Theorem Schu. Let $C$ be a nonempty closed bounded convex subset of a Hilbert space H. Let $L>0$ and $T: C \rightarrow C$ be completely continuous, uniformly L-Lipschitzian and asymptotically pseudo-contractive with sequence $\left\{k_{n}\right\} \subset[1, \infty), q_{n}=2 k_{n}-1$ for all $n \geq 1, \sum_{n=1}^{\infty}\left(q_{n}^{2}-1\right)<\infty,\left\{\alpha_{n}\right\}$ and $\left\{\beta_{n}\right\} \subset[0,1]$, $\epsilon \leq \alpha_{n} \leq \beta_{n} \leq b$ for all $n \geq 1$ and for some $\epsilon>0$ and some $b \in\left(0, L^{-2}\left[\sqrt{1+L^{2}}-1\right]\right)$. For given $x_{1} \in K$, define a sequence $\left\{x_{n}\right\}$ in $C$ by the following algorithm:

$$
\left\{\begin{array}{l}
y_{n}=\left(1-\beta_{n}\right) x_{n}+\beta_{n} T^{n} x_{n}, \\
x_{n+1}=\left(1-\alpha_{n}\right) x_{n}+\alpha_{n} T^{n} y_{n}, \quad \forall n \geq 1 .
\end{array}\right.
$$

Then $\left\{x_{n}\right\}$ converges strongly to some fixed point of $T$. 
Two classical iteration processes are often used to approximate a fixed point of a nonexpansive mapping and its extensions. The first one was introduced by Mann [7], which is defined as follows:

$$
\left\{\begin{array}{l}
x_{0} \in C \quad \text { arbitrary choosen, } \\
x_{n+1}=\left(1-\alpha_{n}\right) x_{n}+\alpha_{n} T x_{n}, \quad \forall n \geq 0,
\end{array}\right.
$$

where $\left\{\alpha_{n}\right\}$ is a sequence in the interval $(0,1)$.

The second one was referred to as Ishikawa iteration process [4], which is defined recursively as follows:

$$
\left\{\begin{array}{l}
x_{0} \in C \quad \text { arbitrary choosen, } \\
y_{n}=\left(1-\beta_{n}\right) x_{n}+\beta_{n} T x_{n}, \\
x_{n+1}=\left(1-\alpha_{n}\right) x_{n}+\alpha_{n} T y_{n}, \quad \forall n \geq 0,
\end{array}\right.
$$

where $\left\{\alpha_{n}\right\}$ and $\left\{\beta_{n}\right\}$ are sequences in the interval $(0,1)$.

But both (1.6) and (1.7) have only weak convergence, in general; see [2] and [19]. Reich [14] shows that, if $E$ is a uniformly convex and has a Fréchet differentiable norm, and the sequence $\left\{\alpha_{n}\right\}$ is such that $\sum_{n=0}^{\infty} \alpha_{n}\left(1-\alpha_{n}\right)=\infty$, then the sequence $\left\{x_{n}\right\}$ generated by the process (1.6) converges weakly to a point in $F(T)$ (an extension of the results to the process (1.7) can be found in [19]). Therefore, many authors have attempted to modify (1.6) and (1.7) to have strong convergence.

In 2006, Martinez-Yanes and Xu 9] modified (1.7) to have strong convergence by hybrid projection algorithms in Hilbert spaces. To be more precise, They proved the following result.

Theorem MYX. Let $C$ be a closed convex subset of a Hilbert space $H$ and $T: C \rightarrow C$ be a nonexpansive mapping such that $F(T) \neq \emptyset$. Assume that $\left\{\alpha_{n}\right\}$ and $\left\{\beta_{n}\right\}$ are sequences in $[0,1]$ such that $\alpha_{n} \leq 1-\delta$ for some $\delta \in(0,1]$ and $\beta_{n} \rightarrow 1$. Define a sequence $\left\{x_{n}\right\}$ in $C$ by the following algorithm:

$$
\left\{\begin{array}{l}
x_{0} \in C \quad \text { chosen arbitrarily, } \\
z_{n}=\beta_{n} x_{n}+\left(1-\beta_{n}\right) T x_{n} \\
y_{n}=\alpha_{n} x_{n}+\left(1-\alpha_{n}\right) T z_{n} \\
C_{n}=\left\{v \in C:\left\|y_{n}-v\right\|^{2} \leq\left\|x_{n}-v\right\|^{2}+\left(1-\alpha_{n}\right)\left(\left\|z_{n}\right\|^{2}-\left\|x_{n}\right\|^{2}+2\left\langle x_{n}-z_{n}, v\right\rangle\right)\right\} \\
Q_{n}=\left\{v \in C:\left\langle x_{0}-x_{n}, x_{n}-v\right\rangle \geq 0\right\} \\
x_{n+1}=P_{C_{n} \cap Q_{n}} x_{0} .
\end{array}\right.
$$

Then $\left\{x_{n}\right\}$ converges in norm to $P_{F(T)} x_{0}$.

Recently, Qin, Su and Shang [13] improved the results of Martinez-Yanes and Xu 9 from nonexpansive mappings to asymptotically nonexpansive mappings. More precisely, They proved the following theorem.

Theorem QSS. Let $C$ be a bounded closed convex subset of a Hilbert space $H$ and $T: C \rightarrow C$ be an asymptotically nonexpansive mapping with a sequence $\left\{k_{n}\right\}$ such that $k_{n} \rightarrow 1$ as $n \rightarrow \infty$. Assume that $\left\{\alpha_{n}\right\}$ is a sequence in $(0,1)$ such that $\alpha_{n} \leq 1-\delta$ for all $n$ and for some $\delta \in(0,1]$ and $\beta_{n} \rightarrow 1$. Define a sequence $\left\{x_{n}\right\}$ in $C$ by the following algorithm:

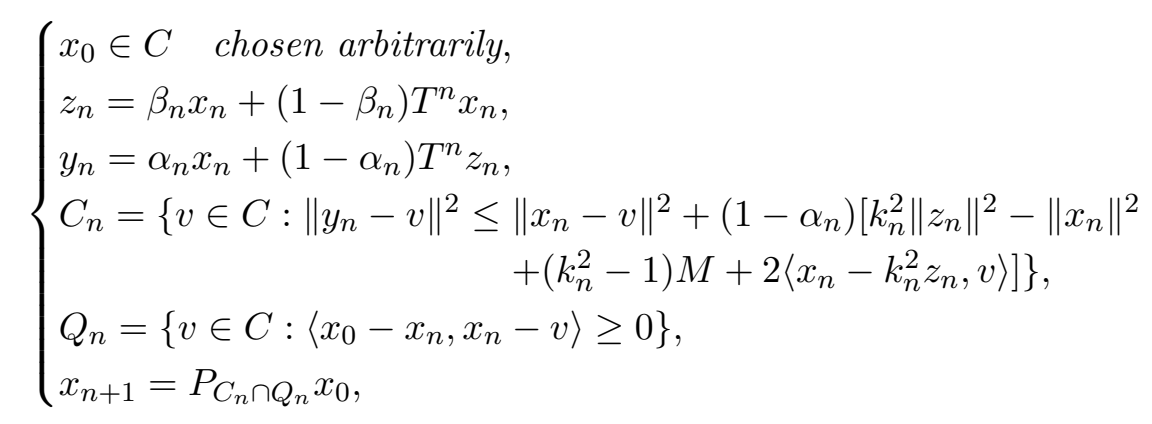

where $M$ is a appropriate constant such that $M>\|v\|^{2}$ for each $v \in C_{n}$, then $\left\{x_{n}\right\}$ converges to $P_{F(T)} x_{0}$. 
Very recently, Zhou [20] improved the results of Martinez-Yanes and Xu [9] from nonexpansive mappings to Lipschitz pseudo-contractions. To be more precise, he proved the following theorem.

Theorem Zhou. Let $C$ be a closed convex subset of a real Hilbert space $H$ and $T: C \rightarrow C$ be a Lipschitz pseudo-contraction such that $F(T) \neq \emptyset$. Suppose that $\left\{\alpha_{n}\right\}$ and $\left\{\beta_{n}\right\}$ are two real sequences in $(0,1)$ satisfying the conditions:

(a) $\beta_{n} \leq \alpha_{n}, \forall n \geq 0$;

(b) $\liminf _{n \rightarrow \infty} \alpha_{n}>0$;

(c) $\limsup _{n \rightarrow \infty} \alpha_{n} \leq \alpha \leq \frac{1}{\sqrt{1+L^{2}}+1}, \forall n \geq 0$, where $L \geq 1$ is the Lipschitzian constant of $T$.

Let a sequence $\left\{x_{n}\right\}$ generated by

$$
\left\{\begin{array}{l}
x_{0} \in C, \\
y_{n}=\left(1-\alpha_{n}\right) x_{n}+\alpha_{n} T x_{n}, \\
z_{n}=\left(1-\beta_{n}\right) x_{n}+\beta_{n} T y_{n}, \\
C_{n}=\left\{z \in C:\left\|z_{n}-z\right\|^{2} \leq\left\|x_{n}-z\right\|^{2}-\alpha_{n} \beta_{n}\left(1-2 \alpha_{n}-L^{2} \alpha_{n}^{2}\right)\left\|x_{n}-T^{n} x_{n}\right\|^{2}\right\}, \\
Q_{n}=\left\{z \in C:\left\langle x_{n}-z, x_{0}-x_{n}\right\rangle \geq 0\right\}, \\
x_{n+1}=P_{C_{n} \cap Q_{n}} x_{0} .
\end{array}\right.
$$

Then $\left\{x_{n}\right\}$ converges strongly to a fixed point $v$ of $T$, where $v=P_{F(T)} x_{0}$.

In this paper, motivated by Acedo and $\mathrm{Xu}$ [1], Kim and $\mathrm{Xu}$ [5, 6], Marino and $\mathrm{Xu}$ [8, Martinez-Yanes and Xu [9], Nakajo and Takahashi [10, Qin et al. [11], Qin, Cho and Zhou [12], Qin, Su and Shang [13], Su and Qin [17, 18] and Zhou [20, 21], we modify Ishikawa iterative process (1.7) to have strong convergence for asymptotically quasi-pseudocontractive mappings in the framework of Hilbert spaces without any compact assumption.

In order to prove our main results, we need the following lemmas.

Lemma 1.1. ([8]) Let $H$ be a real Hilbert space. Then the following equations hold:

(a) $\|x-y\|^{2}=\|x\|^{2}-\|y\|^{2}-2\langle x-y, y\rangle$ for all $x, y \in H$.

(b) $\|t x+(1-t) y\|^{2}=t\|x\|^{2}+(1-t)\|y\|^{2}-t(1-t)\|x-y\|^{2}$ for all $t \in[0,1]$ and $x, y \in H$.

Lemma 1.2. Let $C$ be a closed convex subset of real Hilbert space $H$ and $P_{C}$ be the metric projection from $H$ onto $C$ (i.e., for $x \in H, P_{C} x$ is the only point in $C$ such that $\left\|x-P_{C} x\right\|=\inf \{\|x-z\|: z \in C\}$ ). Given $x \in H$ and $z \in C, z=P_{C} x$ if and only if there holds the relations: $\langle x-z, y-z\rangle \leq 0$ for any $y \in C$.

The following lemma can be found in Zhou and Su [22, we still give the proof for the completeness of the paper.

Lemma 1.3. Let $C$ be a nonempty bounded closed convex subset of $H$ and $T: C \rightarrow C$ be a uniformly L-Lipschitzian and asymptotically quasi-pseudocontractive mapping. Then $F(T)$ is a closed convex subset of $C$.

Proof. From the continuity of $T$, we can conclude that $F(T)$ is closed.

Next, we show that $F(T)$ is convex. If $F(T)=\emptyset$, then the conclusion is always true. Let $p_{1}, p_{2} \in F(T)$. We prove $p \in F(T)$, where $p=t p_{1}+(1-t) p_{2}$, for $t \in(0,1)$. Put $y_{(\alpha, n)}=(1-\alpha) p+\alpha T^{n} p$, where $\alpha \in\left(0, \frac{1}{1+L}\right)$. 
For all $w \in F(T)$, we see that

$$
\begin{aligned}
& \left\|p-T^{n} p\right\|^{2} \\
& =\left\langle p-T^{n} p, p-T^{n} p\right\rangle \\
& =\frac{1}{\alpha}\left\langle p-y_{(\alpha, n)}, p-T^{n} p\right\rangle \\
& =\frac{1}{\alpha}\left\langle p-y_{(\alpha, n)}, p-T^{n} p-\left(y_{(\alpha, n)}-T^{n} y_{(\alpha, n)}\right)\right\rangle+\frac{1}{\alpha}\left\langle p-y_{(\alpha, n)}, y_{(\alpha, n)}-T^{n} y_{(\alpha, n)}\right\rangle \\
& =\frac{1}{\alpha}\left\langle p-y_{(\alpha, n)}, p-T^{n} p-\left(y_{(\alpha, n)}-T^{n} y_{(\alpha, n)}\right)\right\rangle+\frac{1}{\alpha}\left\langle p-w+w-y_{(\alpha, n)}, y_{(\alpha, n)}-T^{n} y_{(\alpha, n)}\right\rangle \\
& \leq \frac{1+L}{\alpha}\left\|p-y_{(\alpha, n)}\right\|^{2}+\frac{1}{\alpha}\left\langle p-w, y_{(\alpha, n)}-T^{n} y_{(\alpha, n)}\right\rangle+\frac{1}{\alpha}\left\langle w-y_{(\alpha, n)}, y_{(\alpha, n)}-T^{n} y_{(\alpha, n)}\right\rangle \\
& \leq(1+L) \alpha\left\|p-T^{n} p\right\|^{2}+\frac{1}{\alpha}\left\langle p-w, y_{(\alpha, n)}-T^{n} y_{(\alpha, n)}\right\rangle+\frac{1}{\alpha}\left(k_{n}-1\right)\left\|w-y_{(\alpha, n)}\right\|^{2} .
\end{aligned}
$$

This implies that

$$
\alpha[1-(1+L) \alpha]\left\|p-T^{n} p\right\|^{2} \leq\left\langle p-w, y_{(\alpha, n)}-T^{n} y_{(\alpha, n)}\right\rangle+\left(k_{n}-1\right)\left\|w-y_{(\alpha, n)}\right\|^{2}, \quad \forall w \in F(T) .
$$

Taking $w=p_{i}, i=1,2$ in (1.8), multiplying $t$ and $(1-t)$ on the both sides of (1.8), respectively and adding up, we see that

$$
\alpha[1-(1+L) \alpha]\left\|p-T^{n} p\right\|^{2} \leq\left(k_{n}-1\right)\left\|w-y_{(\alpha, n)}\right\|^{2} .
$$

This shows that $T^{n} p-p \rightarrow 0$ as $n \rightarrow \infty$. Note that $T$ is uniformly $L$-Lipschitzian. It follows that $T^{n+1} p-T p \rightarrow 0$ as $n \rightarrow \infty$. This is, $p \in F(T)$. This completes the proof.

\section{Main Results}

Theorem 2.1. Let $C$ be a nonempty closed convex subset of a real Hilbert space $H$ and $T: C \rightarrow C$ be a uniformly L-Lipschitz and asymptotically quasi-pseudocontractive mapping such that $F(T)$ is nonempty and bounded. Let $\left\{x_{n}\right\}$ be a sequence generated in the following algorithm:

$$
\left\{\begin{array}{l}
x_{0} \in H \quad \text { chosen arbitrarily, } \\
C_{1}=C, \\
x_{1}=P_{C_{1}} x_{0}, \\
y_{n}=\left(1-\alpha_{n}\right) x_{n}+\alpha_{n} T^{n} x_{n}, \\
z_{n}=\left(1-\beta_{n}\right) x_{n}+\beta_{n} T^{n} y_{n}, \\
C_{n+1}=\left\{z \in C_{n}:\left\|z_{n}-z\right\|^{2} \leq\left\|x_{n}-z\right\|^{2}+\beta_{n} \theta_{n}-\alpha_{n} \beta_{n}\left(1-2 \alpha_{n}-L^{2} \alpha_{n}^{2}\right)\left\|x_{n}-T^{n} x_{n}\right\|^{2}\right\}, \\
x_{n+1}=P_{C_{n+1}} x_{0},
\end{array}\right.
$$

where

$$
\theta_{n}=2\left(k_{n}-1\right)\left[2 k_{n}+1+(1+L)^{2}\right]\left(\sup _{z \in F(T)}\left\|x_{n}-z\right\|\right)^{2} \rightarrow 0 .
$$

Assume that the control sequences $\left\{\alpha_{n}\right\}$ and $\left\{\beta_{n}\right\}$ in $(0,1)$ satisfy the restrictions:

(a) $\beta_{n} \leq \alpha_{n}, \forall n \geq 1$;

(b) $\liminf _{n \rightarrow \infty} \alpha_{n}>1$;

(c) $\lim \sup _{n \rightarrow \infty} \alpha_{n} \leq \alpha<\frac{1}{\sqrt{1+L^{2}}+1}, \forall n \geq 0$.

Then the sequence $\left\{x_{n}\right\}$ converges strongly to $P_{F(T)} x_{0}$. 
Proof. We divide the proof into five parts.

Step 1. Show that $C_{n}$ is closed and convex for all $n \geq 1$.

It is obvious that $C_{1}$ is closed and convex. Assume that $C_{m}$ is closed and convex. Next, we show that $C_{m+1}$ is closed and convex for the same $m$. For all $z \in C_{m}$, we see that

$$
\left\|z_{m}-z\right\|^{2} \leq\left\|x_{m}-z\right\|^{2}+\beta_{m} \theta_{m}-\alpha_{m} \beta_{m}\left(1-2 \alpha_{m}-L^{2} \alpha_{m}^{2}\right)\left\|x_{m}-T^{m} x_{m}\right\|^{2}
$$

is equivalent to the following inequality

$$
2\left\langle x_{m}-z_{m}, z\right\rangle \leq\left\|x_{m}\right\|^{2}-\left\|z_{m}\right\|^{2}+\beta_{m} \theta_{m}-\alpha_{m} \beta_{m}\left(1-2 \alpha_{m}-L^{2} \alpha_{m}^{2}\right)\left\|x_{m}-T^{m} x_{m}\right\|^{2} .
$$

This shows that $C_{m+1}$ is closed and convex. We, therefore, obtain that $C_{n}$ is convex for every $n \geq 1$.

Step 2. Show that $F(T) \subset C_{n}, \forall n \geq 1$.

It is obvious that $F(T) \subset C_{1}$. Assume that $F(T) \subset C_{m}$ for some $m$. Next, we show that $F(T) \subset C_{m+1}$ for the same $m$. In view of Lemma 1.1, for all $u \in F(T) \subset C_{m}$, we see from (1.3) that

$$
\begin{aligned}
\left\|z_{m}-u\right\|^{2}= & \left\|\left(1-\beta_{m}\right)\left(x_{m}-u\right)+\beta_{m}\left(T^{m} y_{m}-u\right)\right\|^{2} \\
= & \left(1-\beta_{m}\right)\left\|x_{m}-u\right\|^{2}+\beta_{m}\left\|T^{m} y_{m}-u\right\|^{2}-\beta_{n}\left(1-\beta_{m}\right)\left\|x_{m}-T^{m} y_{m}\right\|^{2} \\
\leq & \left(1-\beta_{m}\right)\left\|x_{m}-u\right\|^{2}+\beta_{m}\left(\left(2 k_{m}-1\right)\left\|y_{m}-u\right\|^{2}+\left\|y_{m}-T^{m} y_{m}\right\|^{2}\right) \\
& -\beta_{m}\left(1-\beta_{m}\right)\left\|x_{m}-T^{m} y_{m}\right\|^{2}
\end{aligned}
$$

and

$$
\begin{aligned}
& \left\|y_{m}-T^{m} y_{m}\right\|^{2} \\
& =\left\|\left(1-\alpha_{m}\right)\left(x_{m}-T^{m} y_{m}\right)+\alpha_{m}\left(T^{m} x_{m}-T^{m} y_{m}\right)\right\|^{2} \\
& =\left(1-\alpha_{m}\right)\left\|x_{m}-T^{m} y_{m}\right\|^{2}+\alpha_{m}\left\|T^{m} x_{n}-T^{m} y_{m}\right\|^{2}-\alpha_{m}\left(1-\alpha_{m}\right)\left\|x_{m}-T^{m} x_{m}\right\|^{2} \\
& \leq\left(1-\alpha_{m}\right)\left\|x_{m}-T^{m} y_{m}\right\|^{2}+L^{2} \alpha_{m}\left\|x_{m}-y_{m}\right\|^{2}-\alpha_{m}\left(1-\alpha_{m}\right)\left\|x_{m}-T^{m} x_{m}\right\|^{2} \\
& \leq\left(1-\alpha_{m}\right)\left\|x_{m}-T^{m} y_{m}\right\|^{2}+\alpha_{m}\left(L^{2} \alpha_{m}^{2}+\alpha_{m}-1\right)\left\|x_{m}-T^{m} x_{m}\right\|^{2} .
\end{aligned}
$$

Note that

$$
\begin{aligned}
\left\|y_{m}-u\right\|^{2}= & \left(1-\alpha_{m}\right)\left\|x_{m}-u\right\|^{2}+\alpha_{m}\left\|T^{m} x_{m}-u\right\|^{2}-\alpha_{m}\left(1-\alpha_{m}\right)\left\|x_{m}-T^{m} x_{m}\right\|^{2} \\
\leq & \left(1-\alpha_{m}\right)\left\|x_{m}-u\right\|^{2}+\alpha_{m}\left(2 k_{m}-1\right)\left\|x_{m}-u\right\|^{2}+\alpha_{m}\left\|x_{m}-T^{m} x_{m}\right\|^{2} \\
& -\alpha_{m}\left(1-\alpha_{m}\right)\left\|x_{m}-T^{m} x_{m}\right\|^{2} \\
\leq & {\left[1+2 \alpha_{m}\left(k_{m}-1\right)\right]\left\|x_{m}-u\right\|^{2}+\alpha_{m}^{2}\left\|x_{m}-T^{m} x_{m}\right\|^{2} . }
\end{aligned}
$$

Substituting (2.2) and (2.3) into (2.1), we arrive at

$$
\begin{aligned}
\left\|z_{m}-u\right\|^{2} \leq & \left(1-\beta_{m}\right)\left\|x_{m}-u\right\|^{2}+\beta_{m}\left(2 k_{m}-1\right)\left[1+2 \alpha_{m}\left(k_{m}-1\right)\right]\left\|x_{m}-u\right\|^{2} \\
& +\left(2 k_{m}-1\right) \alpha_{m}^{2} \beta_{m}\left\|x_{m}-T^{m} x_{m}\right\|^{2}+\alpha_{m} \beta_{m}\left(L^{2} \alpha_{m}^{2}+\alpha_{m}-1\right)\left\|x_{m}-T^{m} x_{m}\right\|^{2} \\
& +\beta_{m}\left(\beta_{m}-\alpha_{m}\right)\left\|x_{m}-T^{m} y_{m}\right\|^{2} \\
\leq & \left(1-\beta_{m}\right)\left\|x_{m}-u\right\|^{2}+\beta_{m}\left(2 k_{m}-1\right)\left[1+2 \alpha_{m}\left(k_{m}-1\right)\right]\left\|x_{m}-u\right\|^{2} \\
& +2\left(k_{m}-1\right) \alpha_{m}^{2} \beta_{m}\left\|x_{m}-T^{m} x_{m}\right\|^{2}+\alpha_{m} \beta_{m}\left(L^{2} \alpha_{m}^{2}+2 \alpha_{m}-1\right)\left\|x_{m}-T^{m} x_{m}\right\|^{2} \\
& +\beta_{m}\left(\beta_{m}-\alpha_{m}\right)\left\|x_{m}-T^{m} y_{m}\right\|^{2} \\
\leq & \left\|x_{m}-u\right\|^{2}+2\left(k_{m}-1\right) \beta_{m}\left[2 \alpha_{m} k_{m}+1-\alpha_{m}+\alpha_{m}^{2}(1+L)^{2}\right]\left\|x_{m}-u\right\|^{2} \\
& +\alpha_{m} \beta_{m}\left(L^{2} \alpha_{m}^{2}+2 \alpha_{m}-1\right)\left\|x_{m}-T^{m} x_{m}\right\|^{2}+\beta_{m}\left(\beta_{m}-\alpha_{m}\right)\left\|x_{m}-T^{m} y_{m}\right\|^{2} \\
\leq & \left\|x_{m}-u\right\|^{2}+2\left(k_{m}-1\right) \beta_{m}\left[2 k_{m}+1+(1+L)^{2}\right]\left\|x_{m}-u\right\|^{2} \\
& +\alpha_{m} \beta_{m}\left(L^{2} \alpha_{m}^{2}+2 \alpha_{m}-1\right)\left\|x_{m}-T^{m} x_{m}\right\|^{2}+\beta_{m}\left(\beta_{m}-\alpha_{m}\right)\left\|x_{m}-T^{m} y_{m}\right\|^{2} .
\end{aligned}
$$


From the condition (a), we obtain that

$$
\left\|z_{m}-u\right\|^{2} \leq\left\|x_{m}-u\right\|^{2}+\beta_{m} \theta_{m}-\alpha_{m} \beta_{m}\left(1-2 \alpha_{m}-L^{2} \alpha_{m}^{2}\right)\left\|x_{m}-T^{m} x_{m}\right\|^{2} .
$$

Therefore, we obtain that $u \in C_{m+1}$. This concludes that $F(T) \subset C_{n}, \forall n \geq 1$.

Step 3. Show that $\left\{x_{n}\right\}$ is a Cauchy sequence in $C$.

In view of $x_{n}=P_{C_{n}} x_{0}$ and $P_{F(T)} x_{0} \in F(T) \subset C_{n}$ for each $n \geq 1$, we see that

$$
\left\|x_{0}-x_{n}\right\| \leq\left\|x_{0}-P_{F(T)} x_{0}\right\| .
$$

This proves that the sequence $\left\{x_{n}\right\}$ is bounded. From $x_{n}=P_{C_{n}} x_{0}$, we see that

$$
\left\langle x_{0}-x_{n}, x_{n}-y\right\rangle \geq 0, \quad \forall y \in C_{n} .
$$

In view of $x_{n+1} \in C_{n+1} \subset C_{n}$, we see that

$$
\begin{aligned}
0 & \leq\left\langle x_{0}-x_{n}, x_{n}-x_{n+1}\right\rangle \\
& =\left\langle x_{0}-x_{n}, x_{n}-x_{0}+x_{0}-x_{n+1}\right\rangle \\
& \leq-\left\|x_{0}-x_{n}\right\|^{2}+\left\|x_{0}-x_{n}\right\|\left\|x_{0}-x_{n+1}\right\|,
\end{aligned}
$$

that is, $\left\|x_{0}-x_{n}\right\| \leq\left\|x_{0}-x_{n+1}\right\|$. This together with the boundedness of $\left\{x_{n}\right\}$ implies that $\lim _{n \rightarrow \infty}\left\|x_{0}-x_{n}\right\|$ exists. By the construction of $C_{n}$, we see that $C_{m} \subset C_{n}$ and $x_{m}=P_{C_{m}} x_{0} \in C_{n}$ for any positive integer $m \geq n$. From $x_{n}=P_{C_{n}} x_{0}$, we see that

$$
\left\langle x_{0}-x_{n}, x_{n}-x_{m}\right\rangle \geq 0
$$

It follows that

$$
\begin{aligned}
\left\|x_{m}-x_{n}\right\|^{2} & =\left\|x_{m}-x_{0}+x_{0}-x_{n}\right\|^{2} \\
& =\left\|x_{m}-x_{0}\right\|^{2}+\left\|x_{0}-x_{n}\right\|^{2}-2\left\langle x_{0}-x_{n}, x_{0}-x_{m}\right\rangle \\
& \leq\left\|x_{m}-x_{0}\right\|^{2}-\left\|x_{0}-x_{n}\right\|^{2}-2\left\langle x_{0}-x_{n}, x_{n}-x_{m}\right\rangle \\
& \leq\left\|x_{m}-x_{0}\right\|^{2}-\left\|x_{0}-x_{n}\right\|^{2} .
\end{aligned}
$$

Letting $m, n \rightarrow \infty$ in (2.6), we have $\lim _{m, n \rightarrow \infty}\left\|x_{n}-x_{m}\right\|=0$. Hence, $\left\{x_{n}\right\}$ is a Cauchy sequence.

Step 4. Show that $T x_{n}-x_{n} \rightarrow 0$ as $n \rightarrow \infty$.

Since $H$ is a Hilbert space and $C$ is closed and convex, we may assume that

$$
x_{n} \rightarrow q \in C \text { as } n \rightarrow \infty .
$$

Next, we show that $q=P_{F(T)} x_{0}$. To end this, we first show that $q \in F(T)$. By taking $m=n+1$ in $(2.6)$, we arrive at

$$
\lim _{n \rightarrow \infty}\left\|x_{n}-x_{n+1}\right\|=0,
$$

In view of $x_{n+1}=P_{C_{n+1}} x_{0} \in C_{n+1}$, we obtain that

$$
\left\|z_{n}-x_{n+1}\right\|^{2} \leq\left\|x_{n}-x_{n+1}\right\|^{2}+\beta_{n} \theta_{n}-\alpha_{n} \beta_{n}\left(1-2 \alpha_{n}-L^{2} \alpha_{n}^{2}\right)\left\|x_{n}-T^{n} x_{n}\right\|^{2} .
$$

On the other hand, we have

$$
\begin{aligned}
\left\|z_{n}-x_{n+1}\right\|^{2} & =\left\|z_{n}-x_{n}+x_{n}-x_{n+1}\right\|^{2} \\
& =\left\|z_{n}-x_{n}\right\|^{2}+2\left\langle x_{n}-z_{n}, x_{n+1}-x_{n}\right\rangle+\left\|x_{n}-x_{n+1}\right\|^{2} .
\end{aligned}
$$

Combining (2.9) with (2.10) and noting that $z_{n}=\left(1-\beta_{n}\right) x_{n}+\beta_{n} T^{n} y_{n}$, we see that

$$
\beta_{n}^{2}\left\|x_{n}-T^{n} y_{n}\right\|^{2}+2 \beta_{n}\left\langle x_{n}-T^{n} y_{n}, x_{n+1}-x_{n}\right\rangle \leq \beta_{n} \theta_{n}-\alpha_{n} \beta_{n}\left(1-2 \alpha_{n}-L^{2} \alpha_{n}^{2}\right)\left\|x_{n}-T^{n} x_{n}\right\|^{2} .
$$


That is,

$$
\beta_{n}\left\|x_{n}-T^{n} y_{n}\right\|^{2}+2\left\langle x_{n}-T^{n} y_{n}, x_{n+1}-x_{n}\right\rangle \leq \theta_{n}-\alpha_{n}\left(1-2 \alpha_{n}-L^{2} \alpha_{n}^{2}\right)\left\|x_{n}-T^{n} x_{n}\right\|^{2} .
$$

It follows that

$$
\alpha_{n}\left(1-2 \alpha_{n}-L^{2} \alpha_{n}^{2}\right)\left\|x_{n}-T^{n} x_{n}\right\|^{2} \leq \theta_{n}-2\left\langle x_{n}-T^{n} y_{n}, x_{n+1}-x_{n}\right\rangle .
$$

From the assumptions on $\left\{\alpha_{n}\right\}$, we can choose $a \in\left(\alpha, \frac{1}{\sqrt{1+L^{2}}+1}\right)$. For such chosen $a$, there exists a positive integer $N \geq 1$ such that $\alpha_{n}<a$ for all $n \geq N$. It follows that $1-2 a-L^{2} a^{2}>0$. On the other hand, one can choose $b \in(0, c)$, where $c=\liminf _{n \rightarrow \infty} \alpha_{n}$. we obtain that $\alpha_{n}>b$ for $n$ large enough. It follows that

$$
b\left(1-2 a-L^{2} a^{2}\right)\left\|x_{n}-T^{n} x_{n}\right\|^{2} \leq \theta_{n}+M\left\|x_{n+1}-x_{n}\right\|
$$

for $n \geq 0$ large enough, where $M=2 \sup _{n \geq 0}\left\{\left\|x_{n}-T^{n} y_{n}\right\|\right\}$. From (2.8), we obtain that

$$
\lim _{n \rightarrow \infty}\left\|x_{n}-T^{n} x_{n}\right\|=0 .
$$

On the other hand, we have

$$
\begin{aligned}
\left\|x_{n}-T x_{n}\right\|= & \left\|x_{n}-x_{n+1}\right\|+\left\|x_{n+1}-T^{n+1} x_{n+1}\right\|+\left\|T^{n+1} x_{n+1}-T^{n+1} x_{n}\right\| \\
& +\left\|T^{n+1} x_{n}-T x_{n}\right\| \\
\leq & \left\|x_{n}-x_{n+1}\right\|+\left\|x_{n+1}-T^{n+1} x_{n+1}\right\|+L\left\|x_{n+1}-x_{n}\right\|+L\left\|T^{n} x_{n}-x_{n}\right\| .
\end{aligned}
$$

From (2.8) and (2.11), we arrive at

$$
\lim _{n \rightarrow \infty}\left\|x_{n}-T x_{n}\right\|=0 .
$$

Step 5. Show that $x_{n} \rightarrow q=P_{F(T)} x_{0}$ as $n \rightarrow \infty$.

Notice that

$$
\begin{aligned}
\|q-T q\| & \leq\left\|q-x_{n}\right\|+\left\|x_{n}-T x_{n}\right\|+\left\|T x_{n}-T q\right\| \\
& \leq(1+L)\left\|q-x_{n}\right\|+\left\|x_{n}-T x_{n}\right\| .
\end{aligned}
$$

It follows from (2.7) and (2.12) that $q \in F(T)$. From (2.4), we see that

$$
\left\langle x_{0}-x_{n}, x_{n}-y\right\rangle \geq 0, \quad \forall y \in F(T) \subset C_{n} .
$$

Taking the limit in (2.13), we obtain that $\left\langle x_{0}-q, q-y\right\rangle \geq 0, \forall y \in F(T)$. In view of Lemma 1.2, we see that $q=P_{F(T)} x_{0}$. This completes the proof.

Remark 2.2. Theorem 2.1 includes Theorem 4.1 of Kim and Xu [6] a as special case. It also improves the results of Kim and Xu [5] and Qin, Su and Shang [13] from asymptotically nonexpansive mappings to asymptotically quasi-pseudocontractive mappings.

For the class of Lipschitz quasi-pseudocontractive mappings, we have from Theorem 2.1 the following result.

Corollary 2.3. Let $C$ be a nonempty closed convex subset of a real Hilbert space $H$ and $T: C \rightarrow C$ be a L-Lipschitz and quasi-pseudocontractive mapping such that $F(T) \neq \emptyset$. Let $\left\{x_{n}\right\}$ be a sequence generated in the following algorithm:

$$
\left\{\begin{array}{l}
x_{0} \in H \quad \text { chosen arbitrarily, } \\
C_{1}=C, \\
x_{1}=P_{C_{1}} x_{0}, \\
y_{n}=\left(1-\alpha_{n}\right) x_{n}+\alpha_{n} T x_{n}, \\
z_{n}=\left(1-\beta_{n}\right) x_{n}+\beta_{n} T y_{n}, \\
C_{n}=\left\{z \in C_{n}:\left\|z_{n}-z\right\|^{2} \leq\left\|x_{n}-z\right\|^{2}-\alpha_{n} \beta_{n}\left(1-2 \alpha_{n}-L^{2} \alpha_{n}^{2}\right)\left\|x_{n}-T x_{n}\right\|^{2}\right\}, \\
x_{n+1}=P_{C_{n+1}} x_{0} .
\end{array}\right.
$$


Assume that the control sequences $\left\{\alpha_{n}\right\}$ and $\left\{\beta_{n}\right\}$ in $(0,1)$ satisfy the restrictions:

(a) $\beta_{n} \leq \alpha_{n}, \forall n \geq 1$;

(b) $\liminf _{n \rightarrow \infty} \alpha_{n}>1$;

(c) $\limsup _{n \rightarrow \infty} \alpha_{n} \leq \alpha<\frac{1}{\sqrt{1+L^{2}}+1}, \forall n \geq 0$.

Then the sequence $\left\{x_{n}\right\}$ converges strongly to $P_{F(T)} x_{0}$.

Remark 2.4. Comparing Corollary 2.3 with Theorem 3.6 of Zhou [20], we do not require that the mapping $I-T$ is demi-closed at zero. From the computation point of view, we remove the iterative step $Q_{n}$, see [20] for more details.

Remark 2.5. Corollary 2.3 also gives an affirmative answer to the problem proposed by Marino and Xu [8].

\section{References}

[1] G.L. Acedo and H.K. Xu, Iterative methods for strict pseudo-contractions in Hilbert spaces, Nonlinear Anal., 67 (2007), 2258-2271. 1

[2] A. Genel and J. Lindenstrass, An example concerning fixed points, Israel J. Math., 22 (1975), 81-86. 1

[3] K. Goebel and W.A. Kirk, A fixed point theorem for asymptotically nonexpansive mappings, Proc. Amer. Math. Soc., 35 (1972), 171-174. 1

[4] S. Ishikawa, Fixed points by a new iteration medthod, Proc. Amer. Math. Soc., 44 (1974), 147-150. 1

[5] T.H. Kim and H.K. Xu, Strong convergence of modified Mann iterations for asymptotically nonexpansive mappings and semigroups, Nonlinear Anal., 64 (2006), 1140-1152. 1, 2.2

[6] T.H. Kim and H.K. Xu, Convergence of the modified Mann's iteration method for asymptotically strict pseudo-contractions, Nonlinear Anal., 68 (2008), 2828-2836. 1, 2.2

[7] W.R. Mann, Mean value methods in iteration, Proc. Amer. Math. Soc., 4 (1953), 506-510. 1

[8] G. Marino and H.K. Xu Weak and strong convergence theorems for strict pseudo-contractions in Hilbert spaces, J. Math. Anal. Appl., 329 (2007), 336-346. 1, 1.1, 2.5

[9] C. Martinez-Yanes and H.K. Xu, Strong convergence of the CQ method for fixed point iteration processes, Nonlinear Anal., 64 (2006), 2400-2411. 1 .

[10] K. Nakajo and W. Takahashi, Strong convergence theorems for nonexpansive mappings and nonexpansive semigroups, J. Math. Anal. Appl., 279 (2003), 372-379. 1

[11] X. Qin, Y.J. Cho, S.M. Kang and M. Shang, A hybrid iterative scheme for asymptotically $k$-strict pseudo-contractions in Hilbert spaces, Nonlinear Anal., 70 (2009), 1902-1911.

[12] X. Qin, Y.J. Cho and H. Zhou, Strong convergence theorems of fixed point for quasi-pseudo-contractions by hybrid projection algorithms, Fixed Point Theory, 11 (2010), 347-354. 1

[13] X. Qin, Y. Su and M. Shang, Strong convergence theorems for asymptotically nonexpansive mappings by hybrid methods, Kyungpook Math. J., 48 (2008), 133-142. 1

[14] S. Reich, Weak convergence theorems for nonexpansive mappings in Banach spaces, J. Math. Anal. Appl., 67 (1979), 274-276. 1 , 2.2

[15] B.E. Rhoades, Comments on two fixed point iteration methods, J. Math. Anal. Appl., 56 (1976), 741-750. 1

[16] J. Schu, Iteration constrction of fixed points of asymptotically nonexpansive mappings, J. Math. Anal. Appl., 158 (1991), 107-413. 1

[17] Y. Su and X. Qin, Strong convergence theorems for asymptotically nonexpansive mappings and asymptotically nonexpansive semigroups, Fixed Point Theory Appl., 2006 (2006), Article ID 96215. 1

[18] Y. Su and X. Qin, Monotone CQ iteration processes for nonexpansive semigroups and maximal monotone operators, Nonlinear Anal., 68 (2008), 3657-3664. 1

[19] K.K. Tan and H.K. Xu, Approximating fixed points of nonexpansive mappings by the Ishikawa iteration process, J. Math. Anal. Appl., 178 (1993), 301-308. 1

[20] H. Zhou, Convergence theorems of fixed points for Lipschitz pseudo-contractions in Hilbert spaces, J. Math. Anal. Appl., 343 (2008), 546-556. 1

[21] H. Zhou, Demiclosedness principle with applications for asymptotically pseudo-contractions in Hilbert spaces, Nonlinear Anal., 70 (2009), 3140-3145. $1,2.4$

[22] H. Zhou and Y. Su, Strong convergence theorems for a family of quasi-asymptotic pseudo-contractions in Hilbert spaces, Nonlinear Anal., 70 (2009), 4047-4052. 1 